\title{
The influence of the distal resection margin length on local recurrence and long- term survival in patients with rectal cancer after chemoradiotherapy and sphincter- preserving rectal resection
}

\author{
Jan Grosek ${ }^{1}$, Vaneja Velenik², Ibrahim Edhemovic ${ }^{3}$, Mirko Omejc ${ }^{1,4}$ \\ ${ }^{1}$ Department of Abdominal Surgery, University Medical Centre Ljubljana, Ljubljana, Slovenia \\ 2 Department of Radiotherapy, Institute of Oncology Ljubljana, Ljubljana, Slovenia \\ ${ }^{3}$ Department of Surgery, Institute of Oncology Ljubljana, Ljubljana, Slovenia \\ ${ }^{4}$ Faculty of Medicine, University of Ljubljana, Ljubljana, Slovenia
}

Radiol Oncol 2017; 51(2): 169-177.

Received 20 February 2016

Accepted 10 April 2016

Correspondence to: Prof. Mirko Omejc, M.D., Ph.D., Department of Abdominal Surgery, University Medical Centre Ljubljana, Zaloška cesta 7, SI-1000 Ljubljana, Slovenia. E mail: mirko.omejc@kclj.si

Disclosure: No potential conflicts of interest were disclosed.

Background. Low recurrence rates and long term survival are the main therapeutic goals of rectal cancer surgery. Complete, margin- negative resection confers the greatest chance for a cure. The aim of our study was to determine whether the length of the distal resection margin was associated with local recurrence rate and long-term survival.

Patients and methods. One hundred and nine patients, who underwent sphincter-preserving resection for locally advanced rectal cancer after preoperative chemoradiotherapy between 2006 and 2010 in two tertiary referral centres were included in the study. Distal resection margin lengths were measured on formalin-fixed, pinned specimens. Characteristics of patients with distal resection margin < $8 \mathrm{~mm}$ (Group I, $\mathrm{n}=27$ ), 8-20 mm (Group II, $\mathrm{n}=31$ ) and > 20 $\mathrm{mm}$ (Group III, $\mathrm{n}=51$ ) were retrospectively analysed and compared. Median (range) follow-up time in Group I was 89 (51-111), in Group II 83 (57-111) and in Group III 80 (45-116) months ( $p=0.326)$, respectively.

Results. Univariate survival analysis showed that distal resection margin length was not statistically significantly associated with overall survival or local recurrence rate ( $p$ > 0.05). In a multiple Cox regression analysis, after adjusting for pathologic $\mathrm{T}$ and $\mathrm{N}$ stage $(\mathrm{yT}, \mathrm{yN})$, distal resection margin length was still not statistically significantly associated with overall survival.

Conclusions. Our study shows that close distal resection margins can be accepted as oncologically safe for sphincter-preserving rectal resections after preoperative chemoradiotherapy.

Key words: rectal cancer; distal resection margin; chemoradiotherapy; local recurrence; survival

\section{Introduction}

Rectal carcinoma is one of the commonest forms of cancer in both men and women in the Western world and the second most common cause of death. Even when disease is still localised and surgical resection is considered curative, survival is approxi- mately $60 \%$ at 5 years and approximately $50 \%$ at 10 years. ${ }^{1}$ Although disseminated disease is the most common cause of death, local recurrence causes severe disabling symptoms, is difficult to treat and is often fatal. ${ }^{2,3}$ Local control (i.e. low recurrence rates) and long term survival are the main therapeutic goals of rectal cancer surgery. Secondary 
therapeutic goals are anal sphincter preservation and preservation of voiding and sexual functions, thus improving the quality of life. Complete, margin-negative resection confers the greatest chance for a cure. ${ }^{4}$ Hence, all the resection margins (proximal, distal, and circumferential) must have no microscopic cancer cell residua. Negative distal resection margin (DRM) is defined as a distance from the distal border of the gross tumour (or scar tissue in patients showing clinically complete response after chemoradiation) and the edge of the distal resection margin, in which no cancer cells are found with microscopic examination.

Historically, the standard guidelines recommended DRM of at least $4-5 \mathrm{~cm}$, which meant that sphincter-preserving rectal resection for low lying rectal cancers was practically non-existent. In 1982, Heald published his monumental work in which he recommended the removal of the entire mesorectum with sharp dissection under direct vision, a technique that became known as total mesorectal excision (TME). This ingenious technique, when done properly (i.e. along the embryologic avascular areolar plane, between the mesorectal fascia propria and the fascia of the pelvic sidewall), is advantageous because in addition to including removal of the mesorectum containing the rectal draining lymph nodes, it also facilitates autonomic nerve preservation. TME optimises the oncological outcome by reducing the local recurrence rate and also preserves the quality of life. ${ }^{5}$

Thus, the previously applicable $5 \mathrm{~cm}$ rule was gradually modified to $2 \mathrm{~cm}$ and later with advances in surgical techniques to $1 \mathrm{~cm}$ rule or even less. ${ }^{6,7}$ Preoperative long course chemoradiotherapy (CRT) using 5-fluorouracil (5-FU) regimen has since emerged as the standard of care for patients with locally advanced lower and middle rectal cancer (LARC) ${ }^{8,9}$ To date, the refinements in management have led to a decrease in local recurrence rates from $25-40 \%$ to less than $6 \%$. Seventy-five percent of local recurrences are detected within two years of diagnosing the primary tumour. Around 20\% to $50 \%$ of patients with local recurrences have isolated recurrent disease without distant metastases. . $^{10,11}$

Several reports have shown that in approximately one fourth of cases $(6.5-58 \%)$ there is a substantial, microscopical distal intramural spread of tumour cells (DIS). Whenever DIS is present, it is limited to within $2 \mathrm{~cm}$ in $95 \%$ of all patients. Rarely does it extend for more than $2 \mathrm{~cm}$ in nonirradiated tumours. When it does, it is associated with advanced disease and poor long-term prognosis even when all resection margins are free of disease. ${ }^{12,13}$
Similarly, not often does DIS extend more than 1 $\mathrm{cm}$ from the distal edge of the gross tumour in rectal cancer patients treated with preoperative CRT. When it does, the clinical course of such patients is usually worse, because they rapidly develop distant metastases or/ and locally recurrent disease, regardless of DRM length. ${ }^{14}$ This finding suggests that tumour biology as opposed to resection margin determines the ultimate outcome. ${ }^{15}$

A positive or close circumferential resection margin is strongly associated with local and metastatic recurrence despite CRT and TME. ${ }^{16,17} \mathrm{By}$ contrast, the association of close DRM and its influence on recurrence and long term survival is less clear, with somewhat conflicting reports. ${ }^{18}$ Many centres around the world, including our own two Tertiary Referral Centres (University Medical Centre Ljubljana, Institute of Oncology Ljubljana) have accepted close (1 cm or even less) DRMs as oncologically safe in an effort to maximize the eligibility of patients for sphincter-preserving rectal resection.

The aim of our study was to find out whether the length of the distal resection margin (DRM) has any influence on local recurrence rate and long-term survival among patients with locally advanced rectal cancer treated with preoperative chemoradiotherapy and sphincter-preserving rectal resection.

\section{Patients and methods}

\section{Patients}

Between January 2006 and December 2010, 109 patients who had undergone preoperative CRT and sphincter-preserving rectal resection at two Slovene Tertiary Referral centres (University Medical Centre Ljubljana and Institute of Oncology Ljubljana) were included in our study. We included patients with histologically confirmed rectal adenocarcinoma, confined to the lower and middle third of the rectum without distant disease (M0). Patients had to have had a stage II or stage III disease, confirmed with magnetic resonance imaging (MRI) of the pelvis. Patients enrolled in the study were not supposed to have previously received radiotherapy, chemotherapy or any targeted therapy for rectal cancer. We excluded patients who had other co-existing malignancies or a malignancy within the last 5 years prior to the enrolment other than non- melanoma skin cancer or in situ carcinoma of the cervix, as well as patients with nonradical operation (either $\mathrm{R} 1$ microscopic residua in 
any of the resection margins or R2 macroscopically seen, gross tumour residua).

Pre-treatment work-up consisted of a complete history, physical examination, complete blood count and serum biochemistry, carcinoembryonic antigen (CEA), chest radiography and ultrasonography or computed tomography (CT) scan of the entire abdomen. MRI was done for primary tumour and nodal staging. After discharge, followup visits were scheduled every 3 months for the first 2 years, every 6 months during the 2.-5. year, and yearly thereafter. Physical examination, CEA determination, colonoscopy, chest radiography and ultrasonography or/ and CT scan of the whole abdomen were performed. Recurrences were confirmed pathologically and/ or by sequential imaging with positron emission tomography or MRI. ${ }^{19}$

Patient data and histological tumour characteristics were prospectively collected. The study itself was retrospective and was approved by the National Ethics Committee (\#61/09/14).

\section{Surgery}

Surgery was performed 6-8 weeks after completion of preoperative CRT. All operations were performed by qualified, experienced colorectal surgeons who performed total mesorectal excision with autonomic nerve preservation as the standard procedure. The option for a temporary ileostomy or colostomy was left to the surgeon's discretion. The anastomoses were performed using circular stapling devices.

\section{Chemoradiotherapy}

Patients received preoperative capecitabine-based CRT. They received a total irradiation dose (TD) of 45 Gy to the pelvis plus 5.4 Gy as a boost to the primary tumour in 1.8 Gy daily fractions over 5.5 weeks. Radiotherapy (RT) was delivered using 15 MV photon beams and four-field box technique, once daily, 5 days per week. All fields were treated daily. Patients were irradiated in a prone position with a full bladder and using a belly board to minimise the exposure of the small bowel.

Chemotherapy was administered concomitantly with RT, started on the first day of RT and finished on the last day of RT. Chemotherapy was continuous throughout the RT period and it consisted of oral capecitabine at a daily dose of $1650 \mathrm{mg} / \mathrm{m}^{2}$, divided into two equal doses given 12 hours apart. One dose was taken 1 hour prior to RT. All patients received adjuvant chemotherapy with capecitabine
$1250 \mathrm{mg} / \mathrm{m}^{2}$ orally twice daily on days 1-14 every 3 weeks; 4 cycles were recommended, beginning 6-8 weeks after surgery.

\section{Pathology}

Distal bowel margins were measured in formalin- fixed, pinned specimens. The distal resection margin length was defined as the closest distance between the distal border of the gross tumour (or scar tissue in patients showing clinically complete response after chemoradiation) and the edge of the distal resection. The cutting edges of doughnuts were not included in these measurements, but were also assessed microscopically.

\section{Statistical analysis}

Differences in categorical variables between study groups were analysed using Chi-square test or likelihood ratio test as appropriate. Differences in numeric variables between groups were investigated using Kruskal-Wallis test. Cox's proportional hazards model was used to test the association between each of the risk factors and local recurrence or overall survival. For overall survival, both univariate and multiple Cox regression models were used, but because of the low number of events, the multiple analysis was restricted to include two possible confounders. Proportional hazard assumption was tested graphically by a loglog plot. Time intervals were calculated from the date of the surgery. The $p$-values $<0.05$ were considered statistically significant. Statistical analysis was performed using the SPSS software program (Version 23.0).

\section{Results}

The study included 109 rectal cancer patients. There were 75 male and 34 female patients with an average age of 63 years (range, 34-83). Average length of hospitalization was 9 days (range, 3-52). Altogether, we registered 8 major complications that required surgical re-intervention. There was no postoperative 30 day mortality. Characteristics of patients with distal resection margin (DRM) < $8 \mathrm{~mm}$ (Group I, $\mathrm{n}=27$ ), 8-20 mm (Group II, $\mathrm{n}=$ 31) and $>20$ (Group III, $\mathrm{n}=51$ ) $\mathrm{mm}$ are shown in Table 1. Groups were comparable regarding all characteristics, except for the stage of the illness. Group III consisted of a higher share $(29.4 \%)$ of patients with $\mathrm{N}$ stage of 2 compared with Group II 
TABLE 1. Clinicopathological features of patients according to distal resection margin (DRM)

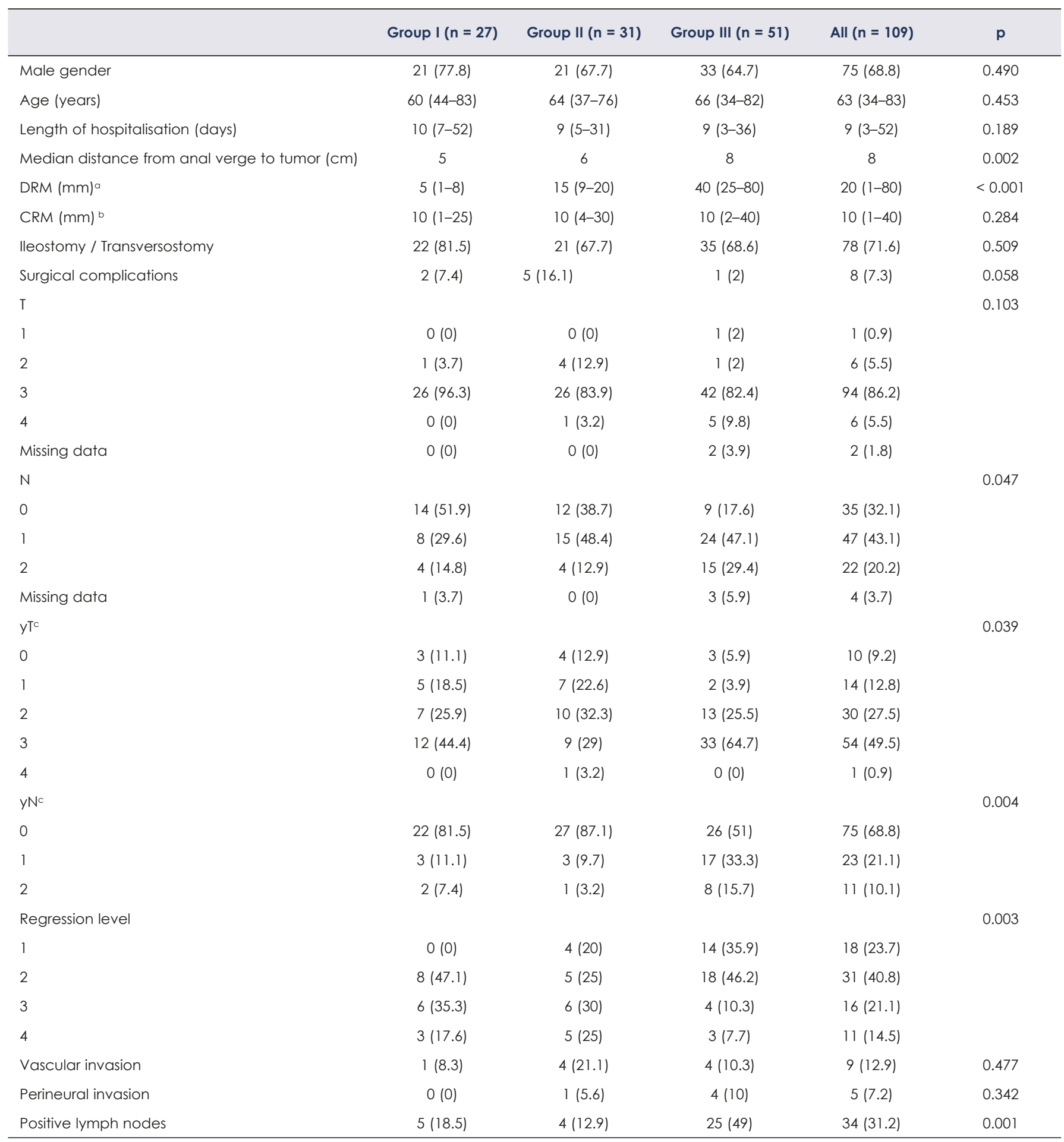

aDRM = distal resection margin; Group I, DRM < 8mm; Group II, $8 \leq$ DRM $\leq 20 \mathrm{~mm}$; Group III, DRM > $20 \mathrm{~mm}$

${ }^{\mathrm{b}} \mathrm{CRM}=$ circumferential resection margin.

${ }^{c} \mathrm{YT}, \mathrm{yN}=$ stage as assessed by pathologic examination of the surgical specimen (after CRT and resection)

Values are shown as median (range) for ordinal and numeric variables and as frequency (percentage) for nominal variable 
TABLE 2. Risk factors for time to local recurrence or death using univariate Cox regression analysis

\begin{tabular}{|c|c|c|c|c|}
\hline Variable (reference group) & Hazard Ratio (95 \% Cl) & P-value & Hazard Ratio (95\% Cl) & P-value \\
\hline Age (years) & $1.1(0.9 ; 1.2)$ & 0.395 & $1(1 ; 1.1)$ & 0.125 \\
\hline Length of hospitalisation (days) & $1.0(0.8 ; 1.2)$ & 0.762 & $1(1 ; 1.1)$ & 0.812 \\
\hline DRM Group III (Group I + Group II) & $3.5(0.4 ; 33.8)$ & 0.276 & $1.4(0.6 ; 3.3)$ & 0.402 \\
\hline $\mathrm{DRM}^{\mathrm{a}}$ & & & & 0.667 \\
\hline DRM Group II ( Group I)a & - & - & $1.3(0.4 ; 4.5)$ & 0.714 \\
\hline DRM Group III (Group I) & - & - & $1.6(0.5 ; 5.1)$ & 0.392 \\
\hline $\mathrm{y}^{\mathrm{c}}$ & - & - & & 0.017 \\
\hline $1(0)$ & - & - & $0.2(0 ; 1.8)$ & 0.15 \\
\hline $2(0)$ & - & - & $0.4(0.1 ; 1.7)$ & 0.215 \\
\hline $3(0)$ & - & - & $0.7(0.2 ; 2.3)$ & 0.517 \\
\hline $4(0)$ & - & - & $19.5(1.6 ; 234.6)$ & 0.019 \\
\hline yN O (1-2) c & $7(0.7 ; 67.2)$ & 0.092 & $2.4(1.1 ; 5.6)$ & 0.040 \\
\hline $\mathrm{yNc}^{\mathrm{N}}$ & - & - & & 0.020 \\
\hline $1(0)$ & - & - & $1.6(0.6 ; 4.6)$ & 0.382 \\
\hline
\end{tabular}

DRM = distal resection margin; Group I, DRM < $8 \mathrm{~mm}$; Group II, $8 \leq \mathrm{DRM} \leq 20 \mathrm{~mm}$; Group III, DRM > 20 mm

${ }^{\mathrm{b}} \mathrm{CRM}=$ circumferential resection margin.

${ }^{c} \mathrm{YT}, \mathrm{yN}=$ stage as assessed by pathologic examination of the surgical specimen (after CRT and resection)

Values are shown as median (range) for ordinal and numeric variables and as frequency (percentage) for nominal variables

$(12.9 \%)$ and Group I $(14.8 \%)(p=0.020)$. After the surgery, a higher share of patients in Group III had a more advanced stage of tumours ( $\mathrm{yT}, \mathrm{p}=0.039$; $\mathrm{yN}, \mathrm{p}=0.004)$ and a lower share had regression levels of 3 and $4(p=0.003)$.

Median (range) follow-up time in Group I was 89 (51-111), in Group II 83 (57-111) and in Group III $80(45-116)$ months $(p=0.326)$, respectively. There were $4(14.8 \%)$ deaths due to rectal cancer in Group I, 6 (19.4\%) in Group II and 12 (23.5\%) in Group III. There were no local recurrences in Group I, 1 in Group II and 3 in Group III. Univariate survival analysis showed DRM length was not statistically significantly associated with overall survival or local recurrence rate $(\mathrm{p}>0.05$; Table 2, Figure 1$)$.

Overall survival was statistically significantly associated with tumour stage after surgery (yT, p $=0.017 ; \mathrm{yN}, \mathrm{p}=0.02)$. Patients with pathologic $\mathrm{T}$ stage 4 (yT4) after the surgery had 19.5 (95\% CI, 1.6-234.6) times higher risk of death than patients with pathologic $\mathrm{T}$ stage 1 (yT1). Patients with pathologic N stage 2 after the surgery (yN2) had 4.1 (95\% CI, 1.5-5.6) times higher risk of death than patients with $\mathrm{N}$ stage 0 (yN0). None of the other risk factors was statistically significantly associated with overall survival. No association between 

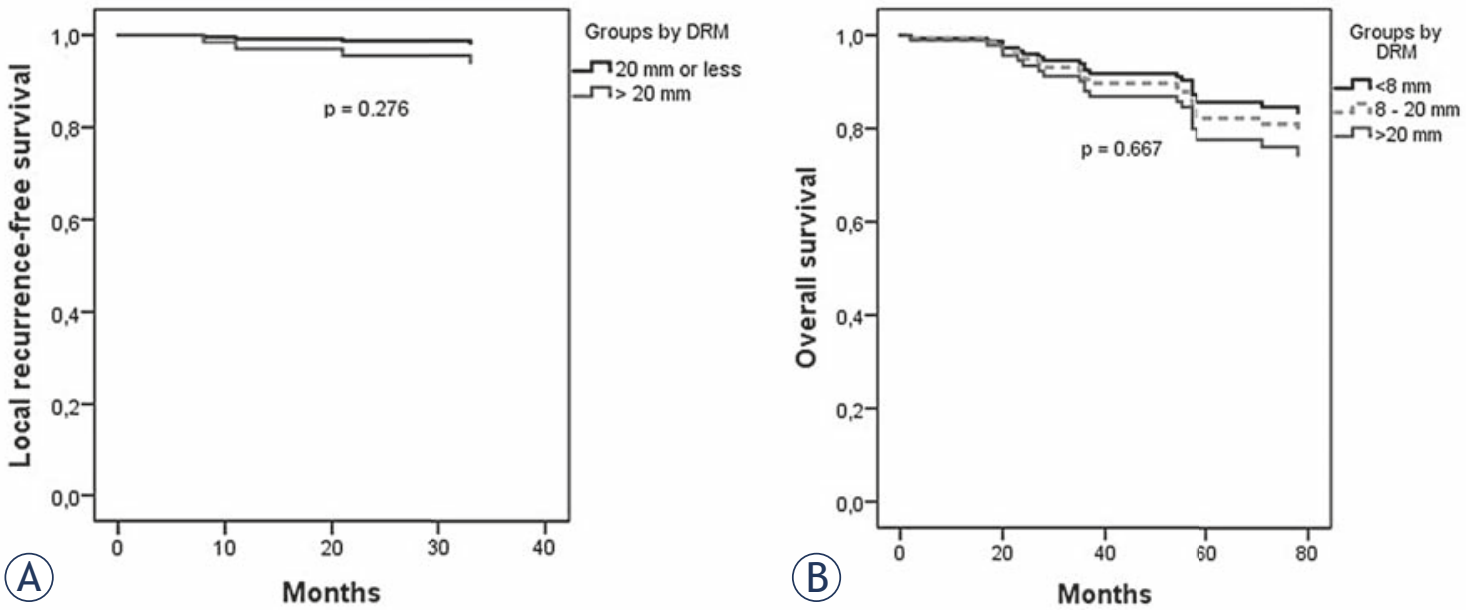

FIGURE 1. Univariate analysis of survival rate: (A) local recurrence-free survival, (B) overall survival rate.

examined risk factors and recurrence-free survival could be found (Table 2, Figure 1).

After adjusting for pathologic stage $\mathrm{T}$ and $\mathrm{N}$ after surgery $(\mathrm{yT}, \mathrm{yN})$, DRM length was still not statistically significantly associated with overall survival. Factors deemed statistically significant in univariate model were also statistically significantly associated with overall survival in multiple survival regression model (Table 3; Figure 2).

\section{Discussion}

The management of locally advanced rectal cancer (T3, T4, and /or N+) is multimodal and is based on preoperative CRT followed by surgery with TME. The latter can be done either as sphincter-preserv-

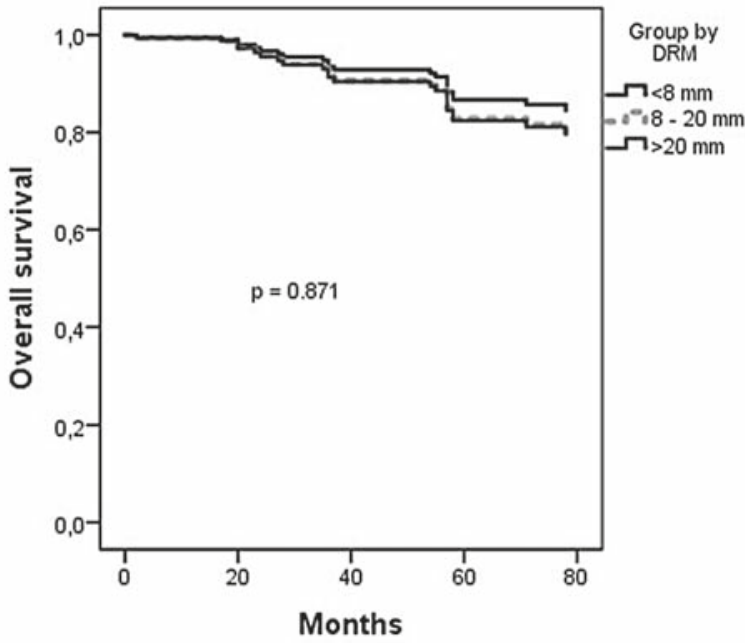

FIGURE 2. Multiple analysis of overall survival rate in the three patient groups. ing low rectal resection or abdominoperineal excision (APE). Preoperative CRT results in downsizing and down-staging of rectal cancer, which often facilitates or even makes possible radical, i.e. R0 resection and thus improves local control. Moreover, the tumour regression can be substantial, in 15$27 \%$ of cases even complete (pathologic complete response) and in such cases sphincter-preserving resections can be done even in cases where primarily APE would be indicated. ${ }^{20,21}$ However, often, regardless of tumour regression and with or without intersphincteric resection a close DRM must be accepted, in order to preserve the anal sphincter. ${ }^{22}$

The present study shows that in patients with rectal cancer after CRT and sphincter-preserving rectal resection, the length of DRM has no statistically significant influence on local recurrence and long-term survival, as long as all the resection margins (proximal, distal, circumferential) have no microscopic cancer cell residua.

Patients in our study were divided into three groups based on the length of the distal resection margins (DRM $<8,8-20$ and $>20 \mathrm{~mm}$, respectively). The cut of values for the subgroups were set theoretically, based on previously published reports. ${ }^{23-28}$ We observed $4(14.8 \%)$ deaths due to rectal cancer in Group I, 6 (19.4\%) in Group II and $12(23.5 \%)$ in Group III. There were no local recurrences in Group I, one in Group II and three in Group III. Univariate survival analysis showed DRM length was not statistically significantly associated with overall survival or local recurrence rate $(p>0.05$; Table 2, Figure 1,2). After adjusting for pathologic stages $\mathrm{T}$ and $\mathrm{N}$ after surgery (yT, $\mathrm{yN})$, the DRM length was still not statistically significantly associated with overall survival (Table 3; 
Figure 3). However, the multiple Cox regression analysis could not be done to the second endpoint of the study (i.e. local recurrence free survival), because there were too few local recurrences.

Our results are supported with several recent reports in the literature. Hong et al. enrolled 218 rectal cancer patients in their study. Patients were classified into three groups according to the length of the DRM $(<1 \mathrm{~cm}, 1-2 \mathrm{~cm},>2 \mathrm{~cm})$. There were no statistically significant differences in survival or local recurrence rate among the groups. A limitation of this study was that histopathologic examination was not conducted properly on the circumferential resection margins in more than half of the enrolled patients. Not all the patients received preoperative CRT and some received postoperative chemoradiotherapy. ${ }^{29}$ This is in contrast to our study, which was a very homogeneous one, as all the enrolled patients uniformly received the same 5-FU based CRT regime. All the specimens were histopathologically tested for all the resection margins, including the circumferential resection margin; if any of the margins were positive, patients were excluded from the study (R1 resection). One of the most comprehensive systematic reviews of the literature was published by Bujko et al. ${ }^{23}$ In this review authors identified 17 studies showing results in relation to margins $<1 \mathrm{~cm}$ versus $>1 \mathrm{~cm}$, five studies in relation to a margin $\leq 5 \mathrm{~mm}$ versus $\geq 5 \mathrm{~mm}$ and five studies showing results in a margin of $\leq 2 \mathrm{~mm}$. Their meta-analysis showed, that in a selected group of patients, DRM $<1 \mathrm{~cm}$ does not jeopardize oncological safety and, furthermore, that even margins shorter than $5 \mathrm{~mm}$ may be acceptable. However, Bujko et al. emphasized, that patient and tumor selection is very important for such an approach. Nevertheless, they could give no precise rules nor specific criteria for such a selection. Apart from this systematic review, there are several other similar reports in literature, either meta-analysis or reports showing results from individual institutions. ${ }^{24-28}$ These reports are more or less heterogeneous with patients being treated with surgery alone or in combination with pre- or postoperative CRT and in these studies there are very different numbers of enrolled patients with different median times of follow-up. In these studies the DRM is analysed either as a continuous variable or as a variable defining cut-off points of different DRM lengths. However, putting the heterogeneity and biases of these reports aside, they generally show no statistically significant differences among different lengths of the DRM on local recurrence rate or on long term survival.
TABLE 3. Risk factors for time to death using multiple Cox regression analysis

\begin{tabular}{lcc}
\hline \multirow{2}{*}{ Variable (reference group) } & \multicolumn{2}{c}{ Overall survival } \\
\cline { 2 - 3 } & Hazard Ratio $(95 \% \mathrm{Cl})$ & P-value \\
\hline $\mathrm{y}^{\mathrm{a}}$ & $0.2(0 ; 2.3)$ & 0.014 \\
$1(0)$ & $0.3(0.1 ; 1.5)$ & 0.212 \\
$2(0)$ & $0.5(0.1 ; 1.9)$ & 0.158 \\
$3(0)$ & $23.1(1.8 ; 302.3)$ & 0.309 \\
$4(0)$ & & 0.017 \\
$\mathrm{yN}^{\mathrm{a}}$ & $1.5(0.4 ; 5.4)$ & 0.034 \\
1 (0) & $4.2(1.4 ; 12.6)$ & 0.489 \\
$2(0)$ & & 0.011 \\
DRM & & 0.871 \\
DRM Group II ( Group I) & $1.3(0.3 ; 5.1)$ & 0.690 \\
DRM Group III (Group I) & $1.4(0.4 ; 4.4)$ & 0.609 \\
\hline
\end{tabular}

a $y T$, yN = stage as assessed by pathologic examination of the surgical specimen (after CRT and resection)

${ }^{\mathrm{b}} \mathrm{DRM}=$ distal resection margin; Group I, DRM < 8mm; Group II, $8 \leq \mathrm{DRM} \leq 20 \mathrm{~mm}$; Group III, DRM $>20 \mathrm{~mm}$

Values are shown as median (range) for ordinal and numeric variables and as frequency (percentage) for nominal variables

By contrast, Vernava et al. reported that DRM $\leq$ $8 \mathrm{~mm}$ statistically significantly worsens both local control and long term survival. ${ }^{30}$ The interpretation of this study was, however, complicated by the fact, that the patients between 1977 and 1985 were treated before the adoption of the TME. ${ }^{31} \mathrm{We}$ believe that strict adherence to TME principles is critical and this is why we believe our group of patients with DRM $<8 \mathrm{~mm}$ did not have statistically significantly worse local recurrence rate or overall survival. None of the 27 patients in group I have had recurrent disease to date (median follow-up 89 months), although there were also minimally negative DRMs in this group of patients (median DRM length $5 \mathrm{~mm} ; 1-8 \mathrm{~mm}$ ). Moreover, univariate analysis of overall survival rate and local recurrence-free survival as well as a multivariate analysis of overall survival rate in the three patient groups, adjusted for $\mathrm{yT}$ and $\mathrm{yN}$ stage, showed a slight but still statistically nonsignificant tendency, that patients from group I (DRM $<8 \mathrm{~mm}$ ) (Figure 1,2) may have had even lower recurrence rates and better long- term survival. Possible explanation for this is that the operating surgeon, due to his experience in rectal cancer, performed APE rather than sphincter-preserving surgery in selected cases with bulky, fixed or otherwise more unfavourable tumours, because he believed that this was the only way to achieve margin negative resection. This hypothesis is supported with our results (Table 1) which show that 
the three groups of DRM lengths (DRM $<8 \mathrm{~mm}$; Group II, $8 \leq \mathrm{DRM} \leq 20 \mathrm{~mm}$; Group III, DRM $>20$ $\mathrm{mm}$ ) are comparable in all characteristics, except for the stage of the illness. Group of patients with DRM $>20 \mathrm{~mm}$ had a higher share of patients with more advanced tumours $(y \mathrm{~T}, \mathrm{p}=0,039 ; \mathrm{yN}, \mathrm{p}=0$, 003 ) and a lower share of patients with regression levels of 3 and $4(p=0.003)$. This can represent a bias in our study, but one that cannot be avoided when the best interest of patients is in question.

Another possible limitation of our study is that the lengths of DRMs were measured on fixed pinned specimens, whereas the $1 \mathrm{~cm}$ rule refers to margins measured by surgeons under fresh anatomically restored ex vivo conditions. ${ }^{32}$ There is no consensus on whether the margins should be measured in fresh or formalin-fixed specimens. Prospective measurements of DRMs with 5 different methods showed that margins were significantly smaller in unpinned than pinned specimens. Although no significant differences were observed in pinned specimens before or after fixation, there was a significant shrinkage after fixation in unpinned specimens. ${ }^{33}$ To avoid this and for consistency, we measured all of the DRM lengths in pinned and then fixed specimens.

Finally, although it is a well-established fact, that sphincter-preserving rectal resection improves quality of life, such improvement of functional results must be objectively measured, preferably through reliable, validated and sensitive instruments (i.e. questionnaires). ${ }^{34}$ Such quality of life assessment was not systematically done for our patients, hence this important end-point of rectal surgery could not be properly investigated in our study.

In summary, our study shows that in patients with rectal cancer after CRT and sphincter-preserving rectal resection, the length of the distal resection margin has no statistically significant influence on local recurrence and long-term survival, as long as all the resection margins (proximal, distal, circumferential) have no microscopic cancer cell residua. Based on our results, taken in context with current reports in literature, we believe it is reasonable to accept short ( $1 \mathrm{~cm}$ or even less) lengths of DRM in order to perform sphincter-preserving rectal resections after CRT, as long as the TME principles are strictly followed.

\section{Acknowledgements}

We wish to thank Ms. Vanja Erculj, statistician who performed all necessary statistical calculations.

\section{References}

1. van Gijn W, Marinjnen CA, Nagtegaal ID, Kranenbarg EM, Putter H, Wiggers $\mathrm{T}$, et al. Preoperative radiotherapy combined with total mesorectal excision for resectable rectal cancer: 12- year follow- up of the multicentre, randomised controlled TME trial. Lancet Oncol 2011; 12: 575-82.

2. Arredondo A, Baixauli J, Beorlegui C, Arbea L, Rodriguez J, Solla JJ, et al. Prognosis factors for recurrence in patients with locally advanced rectal cancer preoperaticely treated with chemoradiotherapy and adjuvant chemotherapy. Dis Colon Rectum 2013; 56: 416-21.

3. Trakarnsanga A, Ithimakin S, Weiser MR. Treatment of locally advanced rectal cancer: Controversies and questions. Word J Gastroenterol 2012; 18: $5521-32$.

4. Zorcolo L, Rosman S, Restivo A, Pisano M, Nigri GR, Fancellu A, Melis MI. Complete pathologic response after combined modality treatment for rectal cancer and long term survival: A meta- analysis. Ann Surg Oncol 2012; 19: 2822-32.

5. Heald RJ, Husband EM, Ryall RD. The mesorectum in rectal cancer surgery: The clue to pelvic recurrence? Br J Surg 1982; 69: 613-6.

6. Williams NS, Dixon MF, Johnston D. Reappraisal of the 5 centimetre rule of distal excision for carcinoma of the rectum: a study of distal intramural spread and of patients survival. Br J Surg 1983; 70: 150-4.

7. Moore HG, Riedel E, Minsk BD, Saltz L, Paty P, Wong D, et al. Adequacy of $1 \mathrm{~cm}$ distal margin after restorative rectal cancer resection with sharp mesorectal excision and preoperative combined- modality therapy. Ann Surg Oncol 2003; 10: 80-5.

8. Velenik V, Oblak I, Anderluh F. Long term results from a randomized phase II trial of neoadjuvant combined- modality therapy for locally advanced rectal cancer. Radiat Oncol 2010; 5: 88-95.

9. Velenik V, Ocvirk J, Music M, Bracko M, Anderluh F, Oblak I, et al. Neadjuvant capecitabine, radiotherapy and bevacizumab (CRAB) in locally advanced rectal cancer: results of an open- label phase II study. Radiat Oncol 2011; 6: $105-12$.

10. Lim KY, Law WL, Liu R, Poon JT, Fan JF, Lo OS. Impact of neoadjuvant treatment on total mesorectal excision for ultra- low rectal cancers. World I Surg 2010; 8: 23-9.

11. Velenik V. Locally recurrent rectal disease: treatment options. Radiol Oncol 2009; 43: 144-51.

12. Williams NS, Dixon MF, Johnston D. Reappraisal of the $5 \mathrm{~cm}$ rule of distal excision for carcinoma of the rectum: a study of distal intramural spread and of patients' survival. Br J Surg 1983; 70: 150-4.

13. Nakagoe T, Yamaguchi E, Tanaka K, Sawai T, Tsuji T, Shibasaki S, et al. Distal intramural spread is an independent prognostic factor for distant metastasis and poor outcome in patients with rectal cancer: A multivariate analysis. Ann Surg Oncol 2003; 10: 163-70.

14. Mezhir JJ, Smith KD, Fichera A, Hart J, Posner MC, Hurst RD. Presence of distal intramural spread after preoperative combined- modality therapy for adenocarcinoma of the rectum: What is now the appropriate distal resection margin? Surgery 2005; 138: 658-64.

15. Park IJ, Kim JC. Adequate lenghth of the distal resection margin in rectal cancer: From the oncological point of view. J Gastrointest Surg 2010; 14: 1331-7.

16. Wibe B, Rendedal PR, Svensson E, Norstein J, Eide TJ, Myrvold HE, et al. Prognostic signifance of the circumferential resection margin following total mesorectal excision for rectal cancer. Br J Surg 2002; 89: 327-34.

17. Trakarnsanga A, Gonen M, Shia J, Goodman KA, Nash GM, Temple LK, et al. What is the signifance of the circumferential margin in locally advanced rectal cancer after neoadjuvant chemoradiotherapy? Ann Surg Oncol 2013. 20: 1179-84.

18. Nash GM, Weiss A, Dasgupta R, Gonen M, Guillem JG, Wong WS. Close distal margin and rectal cancer recurrence after sphicter- preserving rectal resection. Dis Colon Rectum 2010; 53: 1365-73.

19. Velenik V. Post- treatment surveillance in colorectal cancer. Radiol Oncol 2010; 44: 135-41.

20. Maas M, Nelemans PJ, Valentini V, Das P, Roedel C, Kuo L, et al. Long- term outcome in patients with a pathological complete response after chemoradiation with a pathological complete response after chemoradiation for rectal cancer: a pooled analysis of individual patient data. Lancet Oncol 2010; 11: 835-44. 
21. Kim NK, Kim MS, Al- Asari SF. Update and debate issues in surgical treatment of middle and low rectal cancer. J Korean Soc Coloproctol 2012; 28: 230-40.

22. Ludwig K, Kosinski L. How low is low? Evolving approaches to sphinctersparing resection techniques. Semin Radiat Oncol 2011; 21: 185-95.

23. Bujko J, Rutkowski A, Chang GJ, Michalski W, Chmielik E, Kusnierz J. Is the 1 - $\mathrm{cm}$ rule of distal bowel resection margin in rectal cancer based on clinical evidence? A systematic review. Indian J Surg Oncol 2012; 3: 139-46.

24. Kuvshinoff B, Magfoor I, Miedema B, Bryer M, Westgate S, Wilkes J, et al. Distal margin requirements after preoperative chemoradiotherapy for distal rectal carcinomas: are $<$ or $=1 \mathrm{~cm}$ distal margins sufficient? Ann Surg Oncol 2001; 8: 163-9.

25. Fitzgerald TL, Brinkley J, Zervos EE. Pushing the envelope beyond a centimeter in rectal cancer: Oncological implications of close, but negative margins. J Am Coll Surg 2011; 213: 589-95.

26. Rutkowski A, Nowacki MP, Chwalinski M, Oledzki J, Liszka-D P, Gornicki A, et al. Acceptance of a 5- $\mathrm{mm}$ distal bowel resection margin for rectal cancer: is it safe? Colorectal Dis 2011; 14: 71-8.

27. Komori K, Kanemitsu, Y, Ishiguro S, Shimizu Y, Sano T, Ito S, et al. Adequate lenght of the surgical distal resection margin in rectal cancer: from the viewpoint of pathological findings. Am J Surg 2012; 204: 474-80.

28. Pahlman L, Bujko K, Rutkowski, Michalski W. Altering the therapeutic paradigm towards a distal bowel margin of $<1 \mathrm{~cm}$ in patients with low- lying rectal cancer: a systematic review and commentary. Colorectal Dis 2013 15: $166-74$

29. Hong KS, Monn N, Chung SS, Lee R, Kim KH. Oncological outcomes in rectal cancer with close distal resection margins: a retrospective analysis. Ann Surg Treat Res 2015; 89: 23-9.

30. Vernava AM, Moran M, Rothenberger DA, Wong WD. A prospective evaluation of distal margins in carcinoma of the rectum. Surg Gynecol Obstet 1992; 175: 333-6.

31. Kwak JY, Kim CW, Lim SB, Yu CS, Kim TW, Kim JH, et al. Oncologically safe distal resection margins in rectal cancer patients treated with chemoradiotherapy. J Gastrointest Surg 2012; 16: 1947-54.

32. Nelson H, Pettreli N, Carlin A, Couture J, Fleshman J, Guillem J, et al. Guidelines 2000 for colon and rectal surgery. J Natl Cancer Inst 2001; 93: $583-96$

33. Sondenaa K, Kjellevold KH. A prospective study of the length of the distal margin after low anterior resection for rectal cancer. Int J Colorectal Dis 1990; 5: 103-5.

34. Fazio VW, Zutshi M, Remzi FH, Parc Y, Ruppert R, Furst A, et al. A randomized multicenter trial to compare long- term functional outcome, quality of life, and complications of surgical procedures for low rectal cancers. Ann Surg 2007; 246: 481-90 